\title{
Goal and error training methods in the learning of a positioning response
}

\author{
JEFFERSON L, SULZER, NEWCOMB COLLEGE, TULANE UNIVERSITY
}

C. MICHAEL LEVY, UNIVERSITY OF FLORIDA

Three hundred twenty Ss were given 2 Training trials with one of 3 Goal Training Methods or 5 Error Training Methods prior to 6 Test trials with no IF. The Error trained Ss performed consistently worse during Testing, suggesting that the widely used Error Training techniques are not necessarily the most efficacious.

The purpose of the present investigation was to compare positioning accuracy and performance stability in a discrete motor task following minimal training under a variety of error and goal training conditions. Error training in a positioning task requires $S$ to learn a relationship between kinesthetic stimuli and E-reported error associated with different responses. Goal training differs in that $S$ is not required to learn a relationship between kinesthetic stimuli and arbitrary error information, i.e., $S$ is "trained" by exposing him to the goal response without allowing him to compare the standard with a free response until training is completed. Thus, in goal training the emphasis is on the correct response or goal position rather than on the discrepancy between S's response and the criterion.

Given only two training trials, error training was expected to produce poorer initial test performance because the opportunity to learn response-error scale relationships would be severely restricted. Even with a continuous, linear, quantitative error scale $S$ needs a minimum of two trials with informative feedback (IF) to relate the scale to his responses. Since S's third response would represent his second post-IF trial, it seemed likely that this response would be adjusted on the basis of the final error report, and that this situation might produce poor response stability on continued testing without IF. Although goal training, in which no free, active responses are permitted, has not been very successful in the few instances in which it has been used (Lincoln, 1956), the simplicity of the criterion response and the likelihood of transfer from similar reaching and positioning responses supported the expectation that goal trained Ss would be highly successful. Method

Subjects. The sample consisted of 160 male Ss from the U.S. Naval Aviation Medical Center, Pensacola, Florida and 80 males each from Florida State University and the University of Florida. They were run individually in independent testing rooms during routine testing or lecture sessions. As they appeared, Ss were assigned to one of the groups in the order in which they are listed below, i.e., each eight Ss constituted a complete replication.
Apparatus. Manual Lever D, previously described by Sulzer (1963), was used. Essentially, it consists of a shaft-mounted lever housed within a black wooden box. The $\mathrm{S}$ stands on one side of the device and reaches in to grip and move on command. While executing a response neither the lever nor response scale can be seen by $\mathrm{S}$. Lever displacement is measured in angular degrees. The origin was located 44 degrees above the horizontal, and the largest possible response was 160 degrees. A plate with holes drilled at each degree of arc allowed $\mathrm{E}$ to stop the moving pointer and lever at any desired position by inserting a steel punch in the appropriate hole.

Training Methods and Tests. Each $\mathrm{S}$ was shown the proper stance, grip, and response rate, and was given two Training trials by one of the eight methods described below.

Goal Training

1. Goal-Visual Training: $S$ looked at the lever which was positioned at the target. He neither gripped nor moved the lever.

2. Goal-Position Training: S gripped the lever which was positioned at the target. He was notallowed to move the lever.

3. Goal-Guided Training: S moved the lever until E forcibly stopped it at the target.

Error Training

4. Kinesthetic-IF Training: S moved the lever after which he was guided through a response equal to the magnitude of his error. Direction of error was reported verbally by $\mathrm{E}$.

5. Verbal-IF Training: Identical with KinestheticIF except that following each response, direction and magnitude of response error were reported verbally.

6. Error-Position Training: $S$ gripped the lever which was positioned at the place to which the previous Verbal-IF $S$ had moved. Direction and magnitude of error were reported verbally.

7. Error-Visual Training: $S$ looked at the position to which the previous Verbal-IF $S$ had moved on each trial. Direction and magnitude of error were reported verbally as $\mathrm{S}$ looked at each position.

8. Error-Guided Performance: $S$ was guided through the performance of the previous Verbal-IF S, i.e., his ongoing response was forcibly stopped at the place to which the Verbal-IF S had moved on each trial. Direction and magnitude of error were reported verbally after each response.

After two Training trials, all Ss were given six Test trials at the rate of one per $10 \mathrm{sec}$. No IF, cues, or 
comments were provided by $\mathrm{E}$ until the test was completed. The Ss were told that their task on each Test trial was to "move the lever to the target as accurately as you can."

\section{Resulis and Discussion}

An analysis of variance of the absolute error scores (in degrees) was computed for the two samples and eight methods during the six Test trials. It revealed that the Navy Ss learned more during Training than the College Ss $\left(F=8.29, \mathrm{df}=1 / 304, \mathrm{MS}_{\mathrm{e}}=181.60, \mathrm{p}<.01\right.$; the means were 5.68 and 7.45 , respectively) and that there was significant variation among the Methods $\left(\mathrm{F}=3.70, \mathrm{df}=1 / 304, \mathrm{MS}_{\mathrm{e}}=181.60, \mathrm{p}<.01\right)$. Since neither the Test trials main effect nor any of the interactions was significant, it cannot be concluded that differential response stability was achieved during Testing as a result of the different Training Methods.

The mean absolute error scores for the eight methods during Testing are presented in Table 1. A NewmanKeuls test revealed that the three groups receiving Guided Training, while not differing among themselves, performed better than the five groups receiving Error Training $(p<.01)$. The error scores for the latter groups did not differ significantly among themselves.

Goal training of a positioning response is apparently rare in laboratory settings. Bilodeau, Sulzer, \& Levy (1962) and Sulzer \& Levy (1966) stopped S's ongoing response at the goal, but they also provided IF or allowed comparison responses, which classifies their techniques as error training. Lincoln (1956) compared rate learning with full guidance to verbally and kinesthetically provided error, and found poor and erratic performance in the former condition. Goal training of

Table 1. Mean Absolute Error (in degrees) of the Eight Training Conditions for all Test Trials

\begin{tabular}{lll} 
& Training Method & Mean Absolute Error \\
\hline Goal Training & & \\
& Goal-Visual & 3.94 \\
& Goal-Position & 4.89 \\
Error Training & Goal-Guided & 5.10 \\
& & \\
& Kinesthetic-IF & 7.14 \\
& Verbal-IF & 7.32 \\
& Error-Visual & 7.63 \\
& Error-Guided Performance & 8.01 \\
& Error-Position & 8.47 \\
\hline
\end{tabular}

positioning responses is rarely used in non-laboratory environments because it is generally assumed that active responding and some corrective tuition are required to reduce the strength of erroneous response tendencies and/or reinforce the criterion response. I. Bilodeau (1966) maintains that much motor learning consists of correcting for response-goal discrepancies rather than of acquiring precise response tendencies, and, further, that the $\mathrm{S}$ will not acquire a precise response unless forced to by IF. In laboratory studies of positioning utilizing IF,'S usually must learn to execute the criterion response without foreknowledge of the goal locus and without being able to observe the manipulandum or his arm. I. Bilodeau (1966) asserts that these restrictions are desirable in order to (a) minimize transfer from past experience, (b) insure S's dependence upon Emanipulated IF, and (c) increase response susceptibility to other task variables. The same conditions which provide experimental advantages may, however, impose unfortunate restrictions on skill training in nonlaboratory settings.

While error training techniques, typically employed in positioning tasks, produce fairly rapid learning, it must be concluded that they may not necessarily be the most efficacious, especially under conditions of foreshortened training. The superiority of the goal methods in the present study strongly suggests a need for systematic study of this direct form of training which more closely resembles the methodology of psychophysics than that of trial-and-error learning.

\section{References}

Bilodeau, E. A., Sulzer, J. L., \& Levy, C. M. Theory and data on the interrelationships of three factors of memory. Psychol. Monogr., 1962, 76, No. 20 (Whole No. 539).

Bilodeau, Ina MCD. Information feedback. In E. A. Bilodeau (Ed.), Acquisition of skill, New York, Academic Press, 1966. Pp. 255-296.

Lincoln, R. S. Learning and retaining a rate of movement with the aid of kinesthetic and verbal cues. J. exp. Psychol., 1956, 51, 199-204.

Sulzer, J. L. Manual Lever D: a basic psychomotor apparatus for the study of feedback. Percept. mot. Skills, 1963, 16, 859-862.

Sulzer, J. L., \& Levy, C. M. A test for the effect of numerical magnitude of knowledge of results. Percept. mot. Skills, 1966, $22,311-316$.

\section{Note}

1. Supported in part by National Science Foundation Grant G- 13294 to E. A. Bilodeau, principal investigator. 